\title{
Antigenic variation of gonococcal pilin expression in vivo: analysis of the strain FA1090 pilin repertoire and identification of the pils gene copies recombining with pilE during experimental human infection
}

\author{
Terri S. Hamrick, ${ }^{1}$ Jo Ann F. Dempsey, ${ }^{1}$ Myron S. Cohen ${ }^{1,2}$ \\ and Janne G. Cannon ${ }^{1}$ \\ Author for correspondence: Janne G. Cannon. Tel: +1 919966 4774. Fax: +1919962 8103. \\ e-mail: jgc@med.unc.edu
}

1 Department of Microbiology and Immunology, CB\# 7290, 804 Jones Building, University of North Carolina School of Medicine, NC 27599 Chapel Hill, USA

2 Department of Medicine, University of North Carolina School of Medicine, NC 27599, Chapel Hill, USA

\begin{abstract}
Antigenic variation of gonococcal pilin involves a family of variable genes that undergo homologous recombination, resulting in transfer of variant sequences from the pils silent gene copies into the complete pilE expression locus. Little is known about the specific recombination events that are involved in assembling new variant pilin genes in vivo. One approach to understanding pilin variation in vivo is to carry out experimental human infections with a gonococcal strain having a fully characterized repertoire of pilin genes, so that the specific recombination events occurring in vivo can be determined. To this end, the authors cloned, sequenced and mapped the pilin genes of strain FA1090 of Neisseria gonorrhoeae. This strain contains one pilE locus and 19 silent gene copies that are arranged in five pils loci; the pilE locus and four of the pils loci are clustered in a $35 \mathrm{~kb}$ region of the chromosome. The general features of the pilin loci in FA1090 are similar to those in strain MS11, in which the mechanism of pilin variation has been extensively studied. However, none of the silent copy sequences are identical in the two strains, which emphasizes the extreme variability in this gene family among gonococci. Three male volunteers were inoculated with the same variant of strain FA1090 and developed urethritis within 2-4 d. The pilE gene sequences from a total of 23 colonies cultured from the subjects were analysed, determining which pils silent copy donated each portion of the expressed pilE genes. There were 12 different pilin variants, one of which was the original inoculum variant, among the in vivo-expressed pilE gene sequences. The pilE of the inoculum variant was derived entirely from a single silent copy (pils6c1). However, the pilE genes in the majority of the colonies cultured from the infected subjects were chimeras of sequence derived from two or three silent copies. Recombination to generate new pilE sequences involved exchange of single variable minicassettes, multiple minicassettes, entire silent gene copies, or (rarely) recombination within a minicassette.
\end{abstract}

Keywords: Neisseria gonorrhoeae, gonococcus, recombination, human challenge

\section{INTRODUCTION}

The strict human pathogen Neisseria gonorrhoeae has complex mechanisms for constantly altering the anti-

Abbreviations: $m c$, minicassette; PAR, pilin-associated repeat.

The GenBank accession numbers for the sequences reported in this paper are U58840-U58851, U61269, L28978, L28980, L28987 and L28990-L28992. genic nature of many of its surface components, including the type 4 pili that are believed to be responsible for the initial attachment of the organisms to the mucosal epithelium of the human host (reviewed by Nassif \& So, 1995; Nassif et al., 1999). The antigenic type of the pilus changes at a high frequency via changes in the primary amino acid sequence of the major subunit pilin protein. Pilin antigenic variation may play a role in 
immune evasion during gonococcal infection (Zak et al., 1984; Hagblom et al., 1985; Swanson et al., 1987), and undoubtedly contributed to the failure of a pilin vaccine to provide protection against natural infection (Brinton et al., 1982; Boslego et al., 1991). Pilin subunit sequence changes have also been linked to tissue tropism and to variation in adhesiveness of gonococci in in vitro studies, suggesting that expression of different pilins may allow gonococci to colonize different tissues or body sites (reviewed by Nassif \& So, 1995; Seifert, 1996; Nassif et al., 1999).

The gonococcal chromosome generally has one expression locus (pilE) containing a functional pilin gene and multiple silent loci ( $p i l S)$ containing one or more partial pilin gene copies (silent copies) that are truncated at the $5^{\prime}$ end. The pilE gene has a $5^{\prime}$ constant region, a central semivariable $(\mathrm{SV})$ region and a $3^{\prime}$ hypervariable (HV) region. The silent copies contain only the SV and HV regions (reviewed by Nassif \& So, 1995; Seifert, 1996; Nassif et al., 1999). In strain MS11, the only strain in which most of the pilin genes have been characterized, there are 17 silent copies clustered into five pils loci (Haas et al., 1992). The silent copies (or the corresponding regions of pilE) have also been described as containing six variable regions, or minicassettes $(\mathrm{mc})$, bordered by conserved sequence. The minicassette at the $3^{\prime}$ end of a silent copy is designated mc1; mc6 is at the 5' end (Haas \& Meyer, 1986; Haas et al., 1992). Pilin antigenic variation involves $\mathrm{RecA}-$, $\mathrm{RecO}-$ and $\mathrm{RecQ}-$ dependent non-reciprocal recombination between the pils silent copies and the pilE locus (Koomey et al., 1987; Mehr \& Seifert, 1998). As a result of these recombination events, a newly generated pilE gene with previously silent sequences is expressed. The expression locus may receive all or just part of a variant pilS silent copy; the final pilin gene may thus be a chimera of sequences originating from multiple pils loci. The minicassette model predicts that recombination will occur at the conserved regions interspersed between the variable minicassettes (Haas \& Meyer, 1986; Haas et al., 1992). Seifert and coworkers have proposed alternative models for pilin recombination, suggesting that the minicassettes could be further subdivided and reshuffled, with recombinational end points occurring within them (Seifert, 1996; Howell-Adams \& Seifert, 2000).

The potential for variable recombination events leads to an enormous set of possible pilin antigenic types for any given strain. It is not known whether all of those possible types are equally likely to appear in the population of gonococci infecting a human host. There could be mechanistic recombinational constraints operating in vivo that affect which stored pilin sequences are assembled into new pilE coding sequences. In addition, post-recombinational selection pressures might limit the range of possible pilin types, based on efficiency of assembly, adherence, or some as yet unknown pilus function.

The human challenge model of gonococcal urethritis in male volunteers has provided an opportunity to study the importance of pilus expression and pilin antigenic variation in early infection. Nonpiliated colony variants (Kellogg et al., 1963) and a pilE deletion mutant (authors' unpublished results) are reduced in infectivity in the human challenge model. Swanson et al. (1987) showed that antigenic variation occurred during infection of volunteers with strain MS11 by determining the DNA sequence of pilE in isolates cultured from infected subjects. We carried out a similar study using strain FA1090 (Seifert et al., 1994), and found considerable pilin variation in the early stages of infection with this strain. However, without information about the complete pilin gene repertoire of strain FA1090, we could not determine the precise recombinational events occurring in pilE during infection. We have now cloned and sequenced all of the pil loci in strain FA1090 and determined their organization on the chromosome. This information has allowed us to identify the pils silent copies recombining with pilE to generate new expressed pilins during experimental infection of volunteers with this strain and therefore to localize the end points of the recombination events occurring in the pilin gene during human infection.

\section{METHODS}

Bacterial strains. N. gonorrhoeae FA1090 is a serum-resistant, proline-requiring strain of serotype P.IB (Black et al., 1984; Jerse et al., 1994). FA1090 variant A21, which was used for experimental infection of volunteers 1,2 and 62 , is a piliated, Opa-negative variant with three lipooligosaccharide species, one of which binds monoclonal antibody 3F11 (Jerse et al., 1994, and data not shown). The variable regions of the pilE gene of variant A21 are derived entirely from silent copy 1 in pilS6 (pilS6c1). Gonococci were grown on GCB agar (Difco) with the supplements of Kellogg et al. (1963) at $37^{\circ} \mathrm{C}$ in a $5 \%$ $\mathrm{CO}_{2}$ atmosphere. Escherichia coli strain DH5 $\alpha \mathrm{MCR}$ (GibcoBRL) was grown on LB agar with or without $50-100 \mu \mathrm{g}$ ampicillin $\mathrm{ml}^{-1}$.

Cloning and sequencing methods. To clone the pil genes, $\mathrm{N}$. gonorrhoeae strain FA1090 chromosomal DNA was digested with ClaI and separated on a $1 \%$ agarose gel. The $4.3 \mathrm{~kb}$, $3.4-3.2 \mathrm{~kb}, 2.4 \mathrm{~kb}, 1.6 \mathrm{~kb}$ and $0.9 \mathrm{~kb}$ fractions were eluted from the agarose using a Qiaex DNA extraction kit (Qiagen). The $3 \cdot 4-3 \cdot 2 \mathrm{~kb}$, the $2 \cdot 4 \mathrm{~kb}$ and the $0 \cdot 9 \mathrm{~kb}$ fractions were ligated into the AccI site of pUC18 (Gibco-BRL). The $4.3 \mathrm{~kb}$ and the $1.6 \mathrm{~kb}$ fragments were ligated into the ClaI site of pBluescriptSK + (Stratagene). Ligated products were transformed into E. coli DH5 $\alpha$ MCR (Gibco-BRL). Clones were identified by colony blot hybridization (Sambrook et al., 1989) with the Sma/Cla-specific oligonucleotide (see below). The $3.4 \mathrm{~kb}$ fragment containing the pilE locus could not be cloned into these high-copy-number plasmids, presumably due to toxic overexpression of pilin protein, but was successfully cloned into pBR322. The pilE clone was identified by hybridization with the pilE promoter-specific oligonucleotide pilstart (see below). Southern blot analysis of the clones and of FA1090 chromosomal DNA digested with ClaI confirmed that no deletions or gross rearrangements had occurred during the cloning (data not shown).

To sequence the pil loci, nested deletions of each cloned insert were generated using exonuclease III and S1 nuclease 
(Promega Erase-a-Base Kit). DNA was sequenced by the UNC-CH Automated DNA Sequencing Facility on a model 373A DNA Sequencer (Applied Biosystems) using the Taq DyeDeoxy Terminator Cycle Sequencing Kit (Applied Biosystems). The reactions were typically primed with the M13 forward or the M13 reverse oligonucleotide primers. The pilE gene and several regions of the other clones that lacked sufficient overlaps with the nested deletions were sequenced using custom primers. To sequence the pilE clone, pBR322specific oligonucleotides pBR\#1 (5'-AATAGGCGTATCACGAGGCC-3'), pBR\#2 (5'-GTTGCGGCCGTTGAGCACCG-3') and pBR\#3 (5'-CGGTGCCTGACTGCGTTAGC-3') were used as primers, after generating nested deletions. Sequences were analysed using the GCG Sequence Analysis Software Package. All pilin-gene-containing regions of the clones were sequenced on both strands. The FA1090 pilin gene sequences are deposited in GenBank under accession numbers U58846-U58851.

Oligonucleotides. Oligonucleotides were synthesized using the Applied Biosynthesis System (ABI) and were used for Southern hybridization and PCR without further purification. Oligonucleotide pilstart (5'-GAGATAAACGCATAAAATTTCACC-3') was homologous to the promoter region of the pile gene (Seifert et al., 1994; Wainwright et al., 1994). Oligonucleotides cys1c (5'-CGGTAAAATGGTTCTGCGGAC-3') and cys2r (5'-GCAGGTTGACGGCAGGTGC-3') hybridized to the conserved regions $5^{\prime}$ and $3^{\prime}$, respectively, of mc2 in all pilin genes. Oligonucleotide Sma/Cla (5'-TTGCAAGGCGGGCGGGGTCGTCCG-3') was homologous to the Sma/Cla repeat. The TS6 oligonucleotide $\left(5^{\prime}\right.$-CGCCTGTCAGATAAAAACCG-5') hybridized to the region of the PAR (pilin-associated repeat) sequence extending past the Sma/Cla repeat. The RS1' oligonucleotide (5'-CACGCGCCGATTTCAAACACTTCC-3') was a shortened version of the RS1 oligonucleotide of Haas \& Meyer (1986), hybridizing to the RS1 repeat sequence.

The chromosome mapping experiments required DNA probes specific for each of the pil loci of FA1090. The pilstart oligonucleotide (see above) was specific for the pilE locus. Oligonucleotide TS3 (5'-CAGAGAACGGGTCGCGGCAG$\left.3^{\prime}\right)$ was specific for the pils3 locus and hybridized $3^{\prime}$ of the NheI site in that clone. Oligonucleotide TS1 (5'-AACGTCGTCGTCGTTGGCGG-3') was specific for the pilS2 locus, hybridizing to $\mathrm{mc} 2$ in pilS2c3. Oligonucleotide TS2 (5'AAGAAGGAATGCCCGAACCG-3') was specific for pilS7, hybridizing to DNA outside of the pilin coding region. Oligonucleotide TS4 (5'-GAATCACGGCACATGGCCGG$\left.3^{\prime}\right)$ hybridized to several silent copies in the pilS1 locus, and also to pils7 in mc6. Oligonucleotide TS5 (5'-GCGCACCGAAGCCAACGCCG-3') was generated to mc2 of pilS3c3, and also hybridized to silent gene copies in pilS1 and pilS2. Results of Southern blot experiments with TS4 and TS5 were interpreted by comparing them with results obtained with probes with partially overlapping specificities. The probe for the pils6 locus was a clone consisting of pilS6 flanking sequence that was generated when making the nested deletions for sequencing.

Pulsed-field electrophoresis. Gonococcal chromosomal DNA was prepared in agarose blocks (Dempsey et al., 1991) or purified by caesium chloride density-gradient centrifugation (Sambrook et al., 1989). The DNA was digested with restriction endonucleases and resolved by contour-clamped homogeneous electric field electrophoresis, then transferred to BAS membrane (Schleicher and Schuell) and cross-linked as previously described (Dempsey et al., 1991).
DNA hybridization. Southern blots, colony blots and ${ }^{32} \mathrm{P}$ endlabelling of oligonucleotide probes were as described by Sambrook et al. (1989). Hybridization and washing were done at $55^{\circ} \mathrm{C}$ for TS5, TS1, TS3 and Sma/Cla, and at $50{ }^{\circ} \mathrm{C}$ for TS2, TS4 and pilstart. The hybridization solution contained $4 \times$ SSPE, $2 \times$ Denhardt's solution, $20 \mathrm{mM}$ sodium pyrophosphate and $0 \cdot 2 \%$ SDS. Filters were washed twice for $30 \mathrm{~min}$ in a solution of $1 \times \mathrm{SSC}, 0 \cdot 1 \%$ SDS and $5 \mathrm{mM}$ sodium pyrophosphate, and once for $20 \mathrm{~min}$ in $0.5 \times \mathrm{SSC}, 0.1 \% \mathrm{SDS}$ and $5 \mathrm{mM}$ sodium pyrophosphate.

DNA fragments were labelled with ${ }^{32} \mathrm{P}$ by random priming using an oligo-labelling kit (Pharmacia). These probes were hybridized at $60{ }^{\circ} \mathrm{C}$ for Southern blots of pulsed-field gels or $65^{\circ} \mathrm{C}$ for Southern blots of conventional agarose gels in a solution of $6 \times$ SSC, $0.5 \%$ SDS and $5 \times$ Denhardt's solution. Filters were washed once for $5 \mathrm{~min}$ in a solution of $2 \times \mathrm{SSC}$ and $0 \cdot 1 \%$ SDS at room temperature, once for $15 \mathrm{~min}$ in a solution of $2 \times$ SSC and $0 \cdot 1 \%$ SDS at room temperature, and twice for $1 \mathrm{~h}$ in a solution of $0.1 \times$ SSC and $0.5 \%$ SDS at the hybridization temperature.

Experimental human infection. Procedures for recruiting male subjects, obtaining informed consent, carrying out intraurethral inoculation of gonococci, and processing of urethral swab and urine samples have been described (Cohen et al., 1994). Briefly, male subjects between the ages of 18 and 35 gave informed consent and were admitted to the General Clinical Research Center of UNC Hospitals, which is an inpatient research unit. Subjects were inoculated intraurethrally with a suspension of gonococci using a pediatric catheter. They were examined twice daily by a physician and treated with antibiotics immediately upon developing signs or symptoms of infection or at the end of the trial, which lasted 4-6 d. Daily urine specimens were cultured on GCB agar containing VCN inhibitor (Unipath), as were urethral swab specimens obtained when a subject developed a urethral discharge. Colonies growing on the primary isolation plates after $24 \mathrm{~h}$ incubation were picked into $30 \mu \mathrm{l}$ freezer storage medium (FSM) in microtitre wells and stored at $-70{ }^{\circ} \mathrm{C}$ as previously described (Jerse et al., 1994). All protocols were reviewed and approved by the Institutional Review Board and the General Clinical Research Center Advisory Board of the University of North Carolina at Chapel Hill. Subjects were compensated for their participation.

To determine the DNA sequence of pilE from the colonies in FSM, the pilE gene was amplified by PCR using aliquots of the stored colony suspension as the DNA template. The primers were oligonucleotides pilstart and SP3A (Wright et al., 1994). The PCR profile was $94^{\circ} \mathrm{C}$ for $1 \mathrm{~min}, 60^{\circ} \mathrm{C}$ for $1 \mathrm{~min}, 72^{\circ} \mathrm{C}$ for $1 \mathrm{~min}$ for 30 cycles. PCR products were sequenced using the oligonucleotide pilAW (5'-CCTACCAAGACTACACCGCCC-3') as the primer.

\section{RESULTS}

\section{Pilin genes of strain FA1090}

To clone the pil loci of strain FA1090, we took advantage of the conservation of portions of pil coding sequence and of certain repeated elements near gonococcal pil genes. The Sma/Cla repeat is a $65 \mathrm{bp}$ sequence element located at the $3^{\prime}$ end of each ClaI restriction fragment containing a pil locus in strain MS11 (Meyer et al., 1984). Oligonucleotide probes derived from the Sma/ Cla repeat and from pil coding sequence elements in 


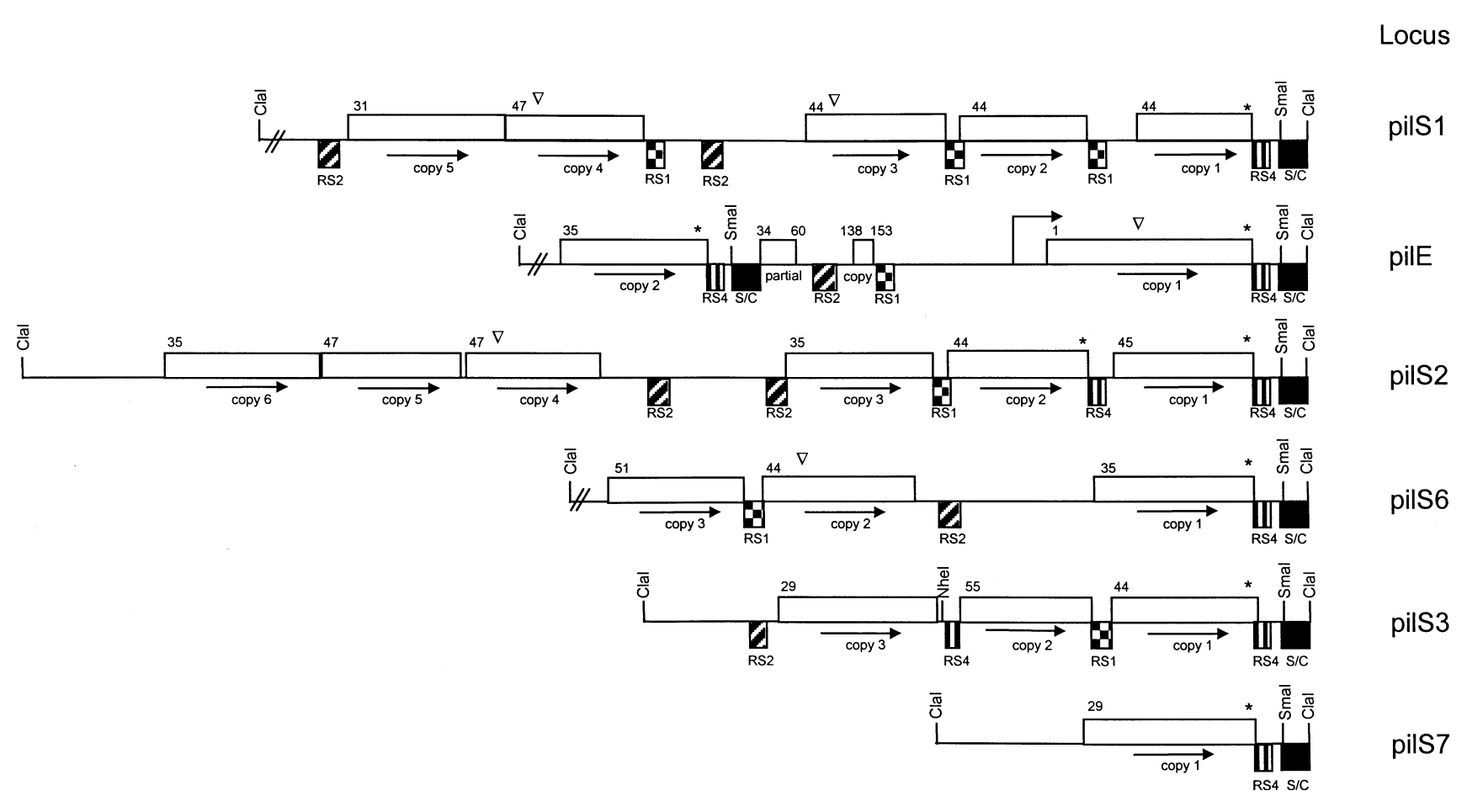

Fig. 1. Schematic representation of $N$. gonorrhoeae strain FA1090 pilin loci. White boxes represent pilus gene copies; the arrows show the orientation of the genes. The $5^{\prime}$ ends of the silent copies are at different positions in mc6; the numbers above the boxes indicate the first amino acid encoded by the translated silent copy, where amino acid 1 is the first amino acid of complete pilin protein. The silent copy at the $3^{\prime}$ end of each pils locus contains a stop codon at its $3^{\prime}$ end (indicated by *); most of the other silent copies lack stop codons. Seven silent copies contain a run of 5-9 cytosine residues in mc5; the gene is out of frame in four of those copies ( $\nabla$ indicates the shift in translational reading frame). Repeated elements: RS2 (49 bp), diagonally striped boxes; RS4 (32 bp), vertically striped boxes; RS1 (39-40 bp), checkered boxes; Sma/Cla (65 bp), black boxes. The silent gene copies that do not contain repeated elements between them overlap or are separated by one nucleotide. The multiple copies of the repeated sequence elements are identical or differ in only a few nucleotides. The pilE locus contains the pilE gene, with a complete pilin coding sequence (starting with amino acid 1) and promoter (bent arrow at $5^{\prime}$ end of the gene). The pilE locus also contains a silent copy plus a partial copy consisting of portions of a pilin gene interrupted by $190 \mathrm{bp}$ of sequence that is unrelated to pilin coding sequence but is similar to pils flanking sequences from FA1090 and from other gonococcal and meningococcal strains. The designations of the pils loci correspond to those in strain MS11 (Bihlmaier et al., 1991; Haas et al., 1987), based on the map locations of the genes.

strain MS11 hybridized to six bands in a Southern blot of ClaI-digested FA1090 DNA; one of those bands also hybridized to the oligonucleotide pilstart, specific for the promoter region of pile (Seifert et al., 1994) (data not shown). We cloned the six pil-containing ClaI fragments and sequenced the pil-containing regions on both strands.

The pilE locus and the five pils loci (pilS1, pilS2, pilS3, pilS6 and pilS7) of strain FA1090 contained a total of 19 silent gene copies (Fig. 1). The general features, conserved domains and organization of the pil loci were similar to those in strain MS11, the only other gonococcal strain for which the pilin gene repertoire has been characterized. However, the number of silent copies in each locus differed in the two strains, as did the number and distribution of the pil-associated repeat elements RS1, RS2 and RS4 (Haas \& Meyer, 1986; Haas et al., 1992). The designations of the FA1090 pils loci correspond to those in strain MS11; we assigned them on the basis of map location of the genes in the two strains (see below).
None of the pilS silent copy sequences were completely duplicated in more than one of the silent copies. In most of the six minicassette regions, all of the silent copies had unique sequences or at most two gene copies had the same sequence. Comparing the 19 sequences at each of the variable minicassette domains showed that regions of sequence similarity were often present in some or all of the silent copies, extending through either the entire minicassette or a portion of it. The $\mathrm{mc} 1$ and $\mathrm{mc} 3$ regions were the least variable, with some versions of these shortest minicassette regions repeated in several of the silent copies. Hypervariable mc2 showed the greatest sequence divergence, although there were a few copies with regions of identical sequence for up to $50 \mathrm{bp}$.

Gonococcal strain MS11 has 17 silent gene copies, 11 of which have been sequenced (Haas \& Meyer, 1986; Haas et al., 1992). None of the sequences from strains FA1090 and MS11 were identical throughout the entire length of a silent gene copy. The two strains shared three versions of mc6, six versions of $\mathrm{mc} 5$, three versions of $\mathrm{mc} 4$, four versions of $\mathrm{mc} 3$ and one version of $\mathrm{mc} 2$. 


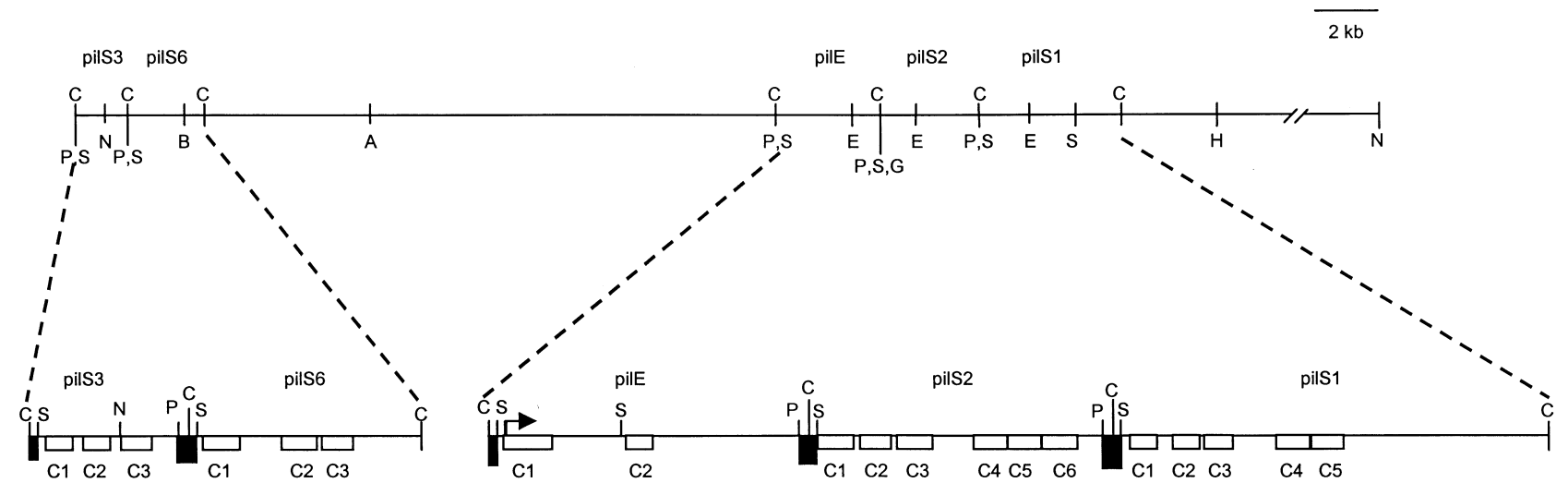

$\overline{300 \mathrm{bp}}$

Fig. 2. Diagram of the major pilin gene cluster, including all but one of the FA1090 pil loci, based on restriction mapping and PCR analysis. Open boxes designate pil gene copies; the different gene copies within each locus are designated C1, $\mathrm{C} 2$, etc. Designations correspond to those in Fig. 1. The orientation of the Clal fragments in this figure is reversed from that in Fig. 1, to be consistent with the arrangement of the loci on the macrorestriction map of the FA1090 chromosome (Dempsey et al., 1991; Dempsey \& Cannon, 1994). The horizontal arrow above copy 1 in the pilE locus indicates that it is the full-length, transcribed pilin gene. All of the other gene copies on the diagram are truncated silent copies. Black boxes at the $3^{\prime}$ end of each pil locus designate the pilin-associated repeat (PAR) sequence, within which is the previously described Sma/Cla repeat. Restriction sites used in mapping: N, Nhel; C, Clal; S, Smal; H, HindIII; P, Pmel; E, EcoRI; G, Bg/ll. The pilS7 locus, which is the only FA1090 pil locus not included on this diagram, is located $\sim 400 \mathrm{~kb}$ from this gene cluster on the chromosome.

\section{Map locations of the FA1090 pil genes}

In previous studies, we constructed a macrorestriction map of the $2200 \mathrm{~kb}$ FA1090 chromosome, using rarecutting restriction enzymes including NheI (Dempsey et al., 1991; Dempsey \& Cannon, 1994). To define the locations of the individual pil loci, we carried out Southern blot analysis of NheI-digested FA1090 chromosomal DNA separated by contour-clamped homogeneous field (CHEF) electrophoresis and probed with oligonucleotides or cloned DNA specific to each pil locus. The pilE, pilS2, pilS6 and pilS1 loci mapped to fragment N10; pilS3, which contains a NheI site within it, mapped to the junction of $\mathrm{N} 10$ and adjacent fragment N4. The pilS7 locus mapped to fragment N6, located $\sim 400 \mathrm{~kb}$ on the map from the major N10/N4 pil gene cluster (data not shown).

To map the relative positions of the loci in the major pil gene cluster, we used a modified Smith-Birnstiel mapping technique (Smith \& Birnstiel, 1976), taking advantage of the NheI site contained in pilS3. FA1090 chromosomal DNA was digested with NheI and with a second enzyme (either partial or complete digests), and the resulting fragments were resolved by conventional or CHEF gel electrophoresis. Southern blots were probed with the pilS3-specific oligonucleotide TS3, which hybridized to fragment N10 near the NheI site, and with DNA probes specific for the other pil loci, to map the positions of the loci within the $62 \mathrm{~kb} \mathrm{~N} 10$ fragment (data not shown). The mapping experiments showed that the pilS3, pilS6, pilE, pilS2 and pilS1 loci were contained in a region of the chromosome less than $35 \mathrm{~kb}$ in size, with pilS3 and pilS6 close together at one end and pilE, pilS2 and pilS1 tightly clustered at the other end (Fig. 2).

The ClaI fragments containing pilS3, pilS2 and pilE, each of which had the Sma/Cla repeat at the $3^{\prime}$ end, also had $98 \%$ identity over 130 bases at their $5^{\prime}$ ends. Through PCR and sequence analysis we determined that the pilS3 and pilS6 loci were on ClaI fragments located next to each other on the FA1090 chromosome and that the $130 \mathrm{bp}$ of repeated sequence at the $5^{\prime}$ end of the pilS3 ClaI fragment was contiguous with the Sma/Cla repeat at the $3^{\prime}$ end of pilS6. Similarly, Southern blot analysis of DNA digested with different restriction enzymes and probed with oligonucleotides specific for the conserved regions at both ends of the pil-containing ClaI fragments confirmed that pilE, pilS2 and pilS1 were contiguous on the FA1090 chromosome, so that the Sma/Cla repeat associated with each locus would be extended by the $130 \mathrm{bp}$ of repeated sequence from the $5^{\prime}$ end of the adjacent ClaI fragment. Examining the sequences of the assembled fragments showed that there was a $265 \mathrm{bp}$ repeated sequence element, containing within it the originally described Sma/Cla and RS4 repeats, 3' of each pil locus (Fig. 3). We designated this conserved region as the PAR (pilin-associated repeat).

\section{Recombination events generating new pilE sequences during experimental human infection}

Experimental gonococcal infection in males results in urethritis that is similar to natural infection in both clinical signs and time course (Cohen et al., 1994). To characterize the recombination events generating new 


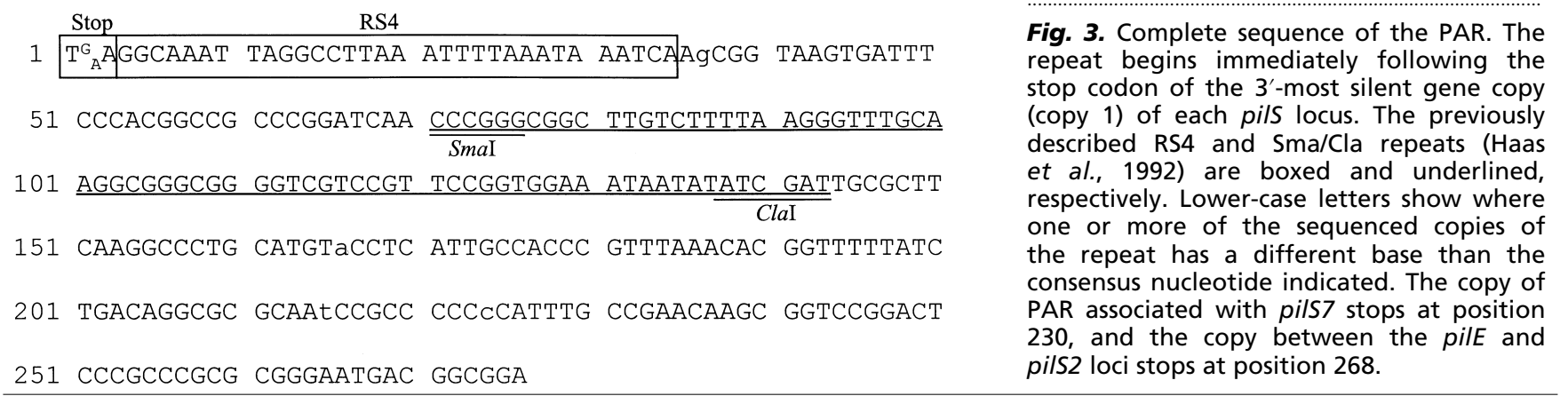

pilin variants in vivo, we inoculated a human volunteer (volunteer 62) intraurethrally with a piliated variant of strain FA1090 (variant A21) in which the predominant expressed pilin sequence was derived entirely from copy 1 in the pilS6 locus (pilS6c1). This variant produces both full-length pilin protein and S-pilin, which is a soluble, truncated form of pilin is that is secreted by some gonococcal variants and is not assembled into pili (Haas et al., 1987). The function of S-pilin, if any, is not known. Variant A21, which was originally isolated in vitro, is not as heavily piliated as most variants that have been recently isolated from patients with gonorrhoea (data not shown). The subject was monitored for development of signs or symptoms of infection, and urine specimens were cultured daily. He was treated as soon as he developed a purulent urethral discharge, which occurred $2 \mathrm{~d}$ after inoculation. Colonies growing on the primary isolation plates from urine or urethral swab specimens all had pilus ${ }^{+}$colony morphology. We amplified and sequenced the pilE gene from 12 colonies that were cultured from this subject at different times. The pilE sequence of one colony that was cultured from a specimen obtained $2 \mathrm{~h}$ after inoculation was identical to that of the inoculum variant. A colony analysed from the first day after inoculation had a portion of the original pilS6c1 sequence replaced with new information. Ten colonies from urine and urethral swab cultures obtained at the time the subject developed a urethral discharge on day 2 had four new pilE sequences among them, in which different portions of the inoculum sequence were replaced. We compared each of the newly expressed pilin sequences to the set of silent copy sequences and identified the silent copy that was the source for each part of the pilE sequence. The expressed sequences were all identical to the relevant portions of the matching silent copy sequences. The recombination end points involved in generating the expressed sequences were localized by finding the point at which an expressed sequence changed from matching one silent copy to matching a different one (Fig. 4).

We carried out similar analyses of the derivation of the in vivo-expressed pilE sequences from our earlier study of two subjects (volunteers 1 and 2) who were also experimentally infected with the FA1090 variant expressing pilE derived entirely from pilS6c1. In that study, we determined the DNA sequence of pilE from individual colonies cultured from the subjects, as well as the pilE sequence from organisms in urethral swab specimens or urine sediments that were frozen without in vitro passage. PCR amplification and sequencing of pilE from the latter specimens had sometimes resulted in multiple bands at some positions in the sequencing gels, although it was usually possible to identify a predominant sequence based on band intensity (Seifert et al., 1994). When we compared the pilE sequences of the reisolates from volunteers 1 and 2 to the pilS storage copies, we could determine unambiguously which pils gene copies were the sources of the expressed sequences only for the seven sequences that had been obtained from colonies growing on the primary isolation plates (Fig. 4). For the sequences obtained from the frozen urine sediment samples or urethral swab specimens, especially those in which a predominant sequence was determined by analysing band intensity in sequencing gels showing mixed sequences, it was difficult to determine a pilS source for all regions of the pilE genes. We therefore confined the analysis to the single-colony isolates from volunteers 1 and 2 that gave unambiguous results in terms of identifying the pils sources for the entire expressed sequences. Other sequences from the earlier study (Seifert et al., 1994) may contain false mosaics or incorrect nucleotides that were assigned based on the subtraction analysis, and should be considered less reliable.

There were 12 unique pilE sequences among the 23 isolates from the three subjects, one the same as the inoculum variant and 11 new sequences (Fig. 4). Information from at least one silent copy in pilS1, pilS2, pilS3 and pilS7 was used to generate one or more pilE sequences in different isolates. Sequence from the silent copy located nearest to pilE on the chromosome did not appear in any of the expressed sequences, and the only pilS6 sequence used was that of the inoculum (pilS6c1). In one isolate from volunteer 2, the entire inoculum pilE sequence was replaced with sequence derived from pilS7c1. In most cases, however, the pilE sequences represented chimeras of minicassettes derived from different pils copies, with recombination occurring at the previously described minicassette borders (Haas \& Meyer, 1986). Many of the pilE sequences retained portions of the pilS6c1-derived inoculum sequence. However, these pilE sequences must have resulted from independent recombination events, since they retained different portions of the original sequence. In two 


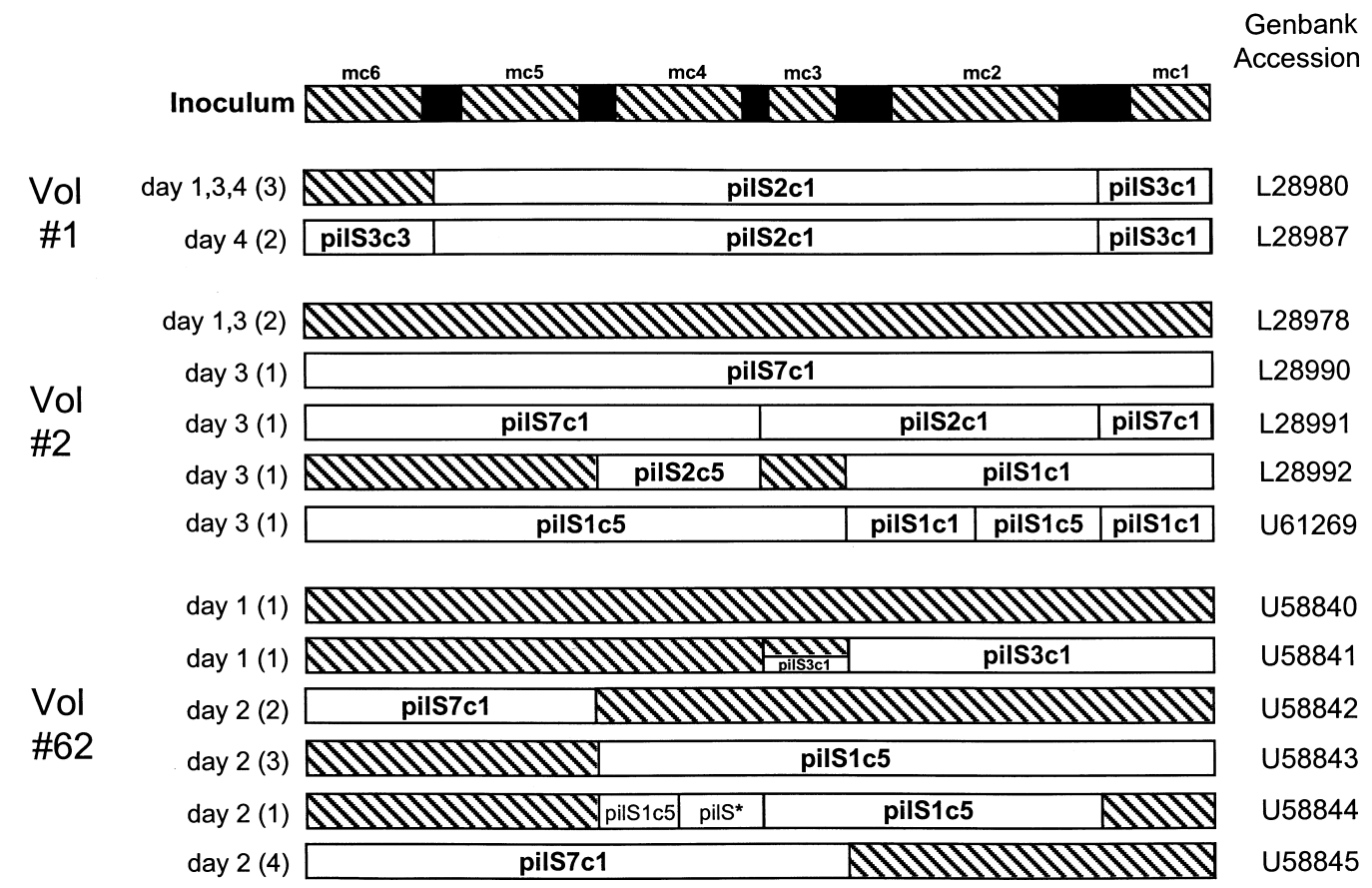

\begin{abstract}
Fig. 4. Diagram of pilE sequences from individual colonies of FA1090 cultured from experimentally infected human subjects. The DNA sequences are identified by their GenBank accession numbers. The pilE sequences were determined for colonies cultured on the primary isolation plates from urine or urethral swab specimens from volunteers 1, 2 and 62. All three subjects were inoculated with a variant of FA1090 expressing pilE derived entirely from silent copy 1 of the pilS6 locus (pilS6c1). Volunteer 1 developed urethritis $3 \mathrm{~d}$ after inoculation; volunteer 2, $4 \mathrm{~d}$ after inoculation; and volunteer $62,2 \mathrm{~d}$ after inoculation. The sequences from volunteers 1 and 2 with accession numbers beginning with the letter $L$ were determined in a previous study (Seifert et al., 1994). The diagrammed sequences do not include the conserved residues encoding amino acids $1-44$ at the $\mathrm{N}$-terminus of pilin protein. Regions of sequence identical to that in the inoculum variant (pilS6c1) are represented by the diagonally striped boxes. All other pils copies are shown as open boxes. Horizontally split boxes indicate regions of sequence that could have been derived from either of two pilS sources. In sequence U58844, the designation pils* in part of mc4 indicates that the sequence could correspond to any of the following silent copies: pilS2c5, pilS2c6, pilS3c1, pilS3c3, pilS6c3, pilS7c1. Recombination end points that are located within the conserved regions flanking the variable minicassettes are drawn in the centre of the conserved regions, although it is not possible to identify the exact end points within those regions. The diagrams were constructed using the minimum number of recombination events required to generate a particular pilin sequence from the different storage copies, and could therefore be an underestimate of the actual amount of recombination that occurred.
\end{abstract}

instances, the only model we could construct to account for the expressed pilE sequence involved recombination occurring within a minicassette. In one isolate from volunteer 2 (U61269), the sequence of mc2 matched pilS1c1 through part of the minicassette and then changed to match pilS1c5. In one of the isolates from volunteer 62 (U58844), recombination appeared to have occurred within mc4, between pilS1c5-derived sequences and DNA from a different silent copy. The other recombination partner might have been any of six different silent copies that have the same sequence in the $3^{\prime}$ portion of mc4.

\section{DISCUSSION}

\section{Intrastrain and interstrain variation in gonococcal pilin sequences}

Despite the intensive effort that has been devoted to studies on the mechanism of gonococcal pilin variation, information about the actual range of intrastrain and interstrain variation in pilin primary sequences is surprisingly limited. Our results showed that the genome of strain FA1090 contained one pilE locus plus 19 silent gene copies organized into five pils loci. The 19 silent copies contained an extensive set of stored variant sequences: none of the silent copy sequences were completely duplicated and most of the variable minicassettes were unique or were shared by at most two silent gene copies. The variable minicassette domains sometimes contained short regions of relatively conserved sequence present in some or all of the silent gene copies. Even hypervariable $\mathrm{mc}$, with the greatest amount of sequence diversity, contained regions up to $50 \mathrm{bp}$ in length that several silent copies had in common. The relative conservation of certain residues within the variable minicassettes in the FA1090 sequences is consistent with the analysis by Seifert (1996), who compared the amino acid sequences of 50 published pilin variants and showed that there are positions with little or no variation located within the originally defined minicassettes. These regions of similarity within otherwise distinct silent copies presumably reflect the evolution of 
the silent copies through a process of gene duplication and subsequent mutation.

The pilE locus and most of the pils loci were clustered in a $35 \mathrm{~kb}$ region of the FA1090 chromosome, with only one pils locus (containing a single silent copy) outside the major cluster. The map positions and sequences of the pil loci match identically to those in the recently completed genome sequence of strain FA1090 (see http://www.genome.ou.edu/gono.html; the GenBank accession number for the completed genome is AE004969). The tight clustering of the pil loci and the numerous DNA repeats surrounding them had the potential to confound the use of shotgun sequencing to assemble the sequence in this region of the gonococcal chromosome. Validating the genome sequencing results with information obtained by a different method increases the reliability of the genome sequence in the region of the chromosome containing these extensively studied genes.

The mapping experiments also revealed a $265 \mathrm{bp}$ repeated sequence at the $3^{\prime}$ end of each of the pils loci (the PAR sequence); the previously described $65 \mathrm{bp} \mathrm{Sma/Cla}$ repeat was actually a portion of this larger repeat, as was a copy of the repeat element RS4. The Sma/Cla repeat, which was proposed by Wainwright et al. (1994) to have a key role in promoting recombination leading to pilin antigenic variation, has homology to previously identified recombinase target sequences. We did not detect any additional homology to those target sequences in the regions of the complete PAR sequence that extend the boundaries of the Sma/Cla element.

The locations of most of the FA1090 pil loci generally corresponded to the mapped positions of the pil loci on the chromosome of strain MS11 (Bihlmaier et al., 1991; Haas et al., 1987). There were, however, some differences in the arrangement and content of the pil genes in the two strains. There is no pil locus in FA1090 corresponding to pilS5 of MS11, which is $180^{\circ}$ across the circular map from the major pil gene cluster (Bihlmaier et al., 1991; Dempsey \& Cannon, 1994). In the mapped variant of strain MS11, a second pilE locus (pilE2) is in the place occupied by pilS3 in FA1090. The two strains had different total numbers of silent copies (19 in FA1090; 17 in MS11); the numbers of silent copies in corresponding pilS loci differed, as did the sequences of the corresponding copies and the identity and arrangement of the repeats flanking them. The differences are consistent with continued rearrangement and mutation of pil genes occurring since the evolutionary divergence of these two strains.

Until now, strain MS11 has been the only one for which extensive pilin sequence information was available (11 of the 17 silent copies) (Haas \& Meyer, 1986; Haas et al., 1992). Comparing the FA1090 sequences with those from MS11 showed that the repertoire of stored pilin information differs in the two strains. None of the silent copies were completely identical in FA1090 and MS11, although a few of the variable minicassettes were the same. There were also short regions of similar or identical deduced amino acid sequence within some variable minicassettes, including the hypervariable mc2, without the exact duplication of the entire minicassette in the two strains. The mc2 sequence that FA1090 and MS11 had in common is not present in all strains (Haas et al., 1992). Since identical mc2 sequences are not present in all strains, as would be expected if this region were a critical functional domain, it is likely that the appearance of the same mc2 sequences in multiple strains is a consequence of frequent horizontal genetic exchange of pilin genes. Haas et al. (1992) estimated that there are no more than approximately 100 different hypervariable mc2 sequences distributed among gonococci. However, since the comparison of the FA1090 and MS11 sequences shows that mc2 regions may contain stretches of sequence that are shared by other silent copies without conservation of an entire minicassette, oligonucleotide hybridization experiments may well underestimate the total number of different sequence variants that exist in the universe of gonococcal strains.

\section{Pilin variation during experimental human infection}

One of the major objectives of this study was to use the information about the pil sequence repertoire of FA1090 to begin characterizing pilin variation occurring during experimental gonococcal infection with this strain. Sequencing the pilE genes in 23 colonies cultured from three experimentally infected volunteers and comparing them to the sequences of the different silent copies showed that at least one copy from each of the pils loci was used for recombination into pile in the 11 newly generated sequences, including the pilS7 locus located $\sim 400 \mathrm{~kb}$ from the major pil gene cluster. There did not appear to be a strong bias towards preferential use of silent copy information from particular pils loci in vivo, although the sample size is relatively small.

In their original model for pilin antigenic variation and recombination, Meyer and coworkers identified the boundaries of the six minicassettes by comparing the DNA sequences of several silent copies and proposed that the end points of recombination between pilS silent copies and pilE would be located within the blocks of absolutely conserved sequence flanking the minicassettes (Haas \& Meyer, 1986; Haas et al., 1992). More recently, Seifert and coworkers have challenged the notion that the variable minicassettes represent the minimal units of coding sequence that could be transferred from a silent copy to the pilE locus by recombination, and have suggested that recombination may occur within the minicassettes (Wainwright et al., 1994, 1997; Mehr \& Seifert, 1998). The great majority of the recombination events that occurred in the in vivo-generated pilin variants in this study involved crossing-over within the conserved blocks of sequence flanking the minicassettes, with transfer of individual minicassettes, multiple minicassettes, or entire pilS gene copies. We did, however, observe two examples of probable intra-minicassette recombination. Comparing the sequences that were involved in the intra-minicassette recombination suggests that 11 base pairs of identity was sufficient for 
pil sequence recombination. Seifert and coworkers have hypothesized that efficient recombination between pil sequences with such limited homology would require specific protein factors in addition to the homologous recombination machinery (Wainwright et al., 1994, 1997; Mehr \& Seifert, 1998).

The pattern of variation in pilin expression in the colonies cultured from volunteer 62 was similar to that in our earlier study of volunteers 1 and 2 (Seifert et al., 1994). Removing from the analysis several sequences that we now realize were less reliable did not alter the conclusions from that earlier study. In all three subjects, new pilin variants appeared early in the infection, with changes occurring throughout the length of the pilE gene. Multiple variants were present simultaneously, although the number of colonies analysed was not sufficient to draw conclusions about the relative proportions of different variants in the infecting population. Volunteer 62 developed urethritis less than $48 \mathrm{~h}$ after inoculation; none of the colonies cultured at that time contained the same pilE gene as the inoculum variant. The 10 colonies analysed from the second day contained four different pilin variants. The culturing of multiple colonies with identical pilE sequences from a specimen suggests that the variants were generated in vivo, and not by switching occurring after isolation of the bacteria from the infected subject. The fact that some of the variants appeared to be recombinants derived from others that appeared earlier in the infection argues that they arose from recombination occurring in vivo (i.e. sequences U58843 and U58844; L28990 and L28991) rather than from clonal expansion of minority variants that were present in the original inoculum. It is not possible to use this type of analysis of pilin variation to determine if the rapid appearance of new pilin variants was a consequence of an elevated rate of variation in vivo, since it is complicated by selection for or against different pilin variants that probably occurred after the actual recombination events.

Gonococcal and meningococcal pilin proteins often contain post-translational modifications, including glycosylation, phosphorylation and modification with glycerophosphate or phosphorylcholine (reviewed by Nassif et al., 1999). In general, these modifications do not appear to have major effects on pilus-mediated adherence to eukaryotic cells in vitro, but they do influence the structure of the pilus fibre and the tendency of the pili to gather into bundles (Virji et al., 1993; Marceau et al., 1998; Jennings et al., 1998; Forest et al., 1999). The function and importance of these pilin modifications in vivo are unknown. The translated sequences of the 19 silent copies in FA1090 differed in some of the amino acid residues that are the potential sites for modification: 11 copies contained serine-63, the site for glycosylation; 18 copies contained serine-68, the site for phosphorylation; 17 copies contained serine-94, the site for modification with $\alpha$-glycerophosphate. Thus, for each of these three modifications, this strain has the potential to express pilin protein that would not be modified, which might be advantageous under particular conditions in vivo. The deduced amino acid sequence of the pilin produced by the pilS6c1-expressing inoculum variant contained an alanine residue at position 63 and serines at positions 68 and 94, and would therefore presumably not be glycosylated but could be phosphorylated and/or modified with $\alpha$-glycerophosphate. In the pilin variants recovered from the volunteers, whenever a new mc6 (the region containing residue 63) was introduced by antigenic variation, it encoded serine-63. However, some colonies retained the inoculum sequence with alanine-63. It would be necessary to follow infected subjects past the time they develop signs of urethritis to determine if the apparent trend towards expression of potentially glycosylated pilins holds true, but there are both ethical and practical impediments to carrying out such experiments.

The rapid shifts in preponderance of different pilin variants in vivo suggest that the infecting population of gonococci was subjected to powerful selective pressures during these early stages of uncomplicated urethral infection in men. Growth of the inoculum variant in vitro did not result in similar shifts in the identity of the predominant variants: after growth of variant A21 on GCB agar for $24 \mathrm{~h}$, more than $98 \%$ of the organisms expressed the original pilS6c1-derived pilin (G. Haskell \& J. G. Cannon, unpublished data). Selection of new pilin variants in vivo was probably not based on evasion of a specific acquired antibody response, given the short duration of the infection. It is possible, however, that immune selection based on pre-existing antibodies or broad non-specific host defences could occur. Alternatively, there may be a selective advantage for gonococci expressing certain pilin types, based on function or on efficiency of pilus assembly. Differences in the primary amino acid sequence of gonococcal or meningococcal PilE influence attachment of the bacteria to different cell types (Lambden et al., 1980; Nassif et al., 1993; Jonsson et al., 1994; Marceau et al., 1995; Marceau \& Nassif, 1999; Long et al., 1998), even though the PilC protein is thought to be the actual adhesin (Rudel et al., 1995; Källström et al., 1997). Sequence differences in PilE may affect adherence indirectly, by changing the structure and properties of the pilus fibre and thus altering the exposure or local density of the PilC adhesin (Nassif et al., 1999). The deduced amino acid sequences of the in vivo-expressed pilins do not provide any clues to the structural basis of such potential functional differences, since the sequences and their silent copy sources were different in the colonies cultured from the three different volunteers. The emergence of different pilin variants in different infected individuals could reflect differences in the binding specificity required for attachment to cellular receptors in those individuals. Alternatively, there may be multiple pilin variants with different primary sequences that can promote the same function. To investigate this question further, we plan to infect subjects twice with the same variant of strain FA1090, characterizing pilin expression in reisolates from both episodes of infection. Such experiments, coupled with in vitro characterization of 
the properties of the pili expressed by in vivo-generated variants, may help in resolving some of the persistent questions surrounding structure-function relationships for gonococcal pilin and the contribution that pilin antigenic variation makes to the success of the gonococcus as a pathogen.

\section{ACKNOWLEDGEMENTS}

We thank Leesa Whicker and Andrea Wallace for technical assistance, Scott Stevens for computer graphics, and Irving Hoffman and the nursing staff of the UNC General Clinical Research Center for their assistance with the clinical aspects of the human infection studies. We also acknowledge the Gonococcal Genome Sequencing Project (supported by USPHS/NIH grant \#AI38399) and B. A. Roe, L. Song, S. P. Lin, X. Yuan, S. Clifton, T. Ducey, L. Lewis and D. W. Dyer at the University of Oklahoma.

This work was supported by grants U01 AI31496 (M.S.C. and J.G.C.) and R01 AI28807 (J.G.C.), and training grant T32 AI07001 (T.S.H.) from the National Institutes of Health.

\section{REFERENCES}

Bihlmaier, A., Romling, U., Meyer, T. F., Tummler, B. \& Gibbs, C. P. (1991). Physical and genetic map of the Neisseria gonorrhoeae strain MS11-N198 chromosome. Mol Microbiol 5, 2529-2539.

Black, W. J., Schwalbe, R. S., Nachamkin, I. \& Cannon, J. G. (1984). Characterization of Neisseria gonorrhoeae protein II phase variation by use of monoclonal antibodies. Infect Immun 45, 453-457.

Boslego, J. W., Tramont, E. C., Chung, R. C. \& 8 other authors (1991). Efficacy trial of a parenteral gonococcal pilus vaccine in men. Vaccine 9, 154-162.

Brinton, C. C., Wood, S. W., Brown, A. \& 7 other authors (1982). The development of a neisserial pilus vaccine for gonorrhea and meningococcal meningitis. In Seminars in Infectious Disease, pp. 140-159. Edited by L. Weinstein \& B. N. Fields. New York: Thieme-Stratton.

Cohen, M. S., Cannon, J. G., Jerse, A. E., Charniga, L., Isbey, S. \& Whicker, L. (1994). Human experimentation with Neisseria gonorrhoeae: rationale, methods, and implications for the biology of infection and vaccine development. J Infect Dis $\mathbf{1 6 9}$, 532-537.

Dempsey, J. A. \& Cannon, J. G. (1994). Locations of genetic markers on the physical map of the chromosome of Neisseria gonorrhoeae FA1090. J Bacteriol 176, 2055-2060.

Dempsey, J. F., Litaker, W., Madhure, A., Snodgrass, T. \& Cannon, J. G. (1991). Physical map of the chromosome of Neisseria gonorrhoeae FA1090 with locations of genetic markers, including opa and pil genes. J Bacteriol 173, 5476-5486.

Forest, K. T., Dunham, S. A., Koomey, M. \& Tainer, J. A. (1999). Crystallographic structure reveals phosphorylated pilin from Neisseria: phosphoserine sites modify type IV pilus surface chemistry and fibre morphology. Mol Microbiol 31, 743-752.

Haas, R. \& Meyer, T. F. (1986). The repertoire of silent pilus genes in Neisseria gonorrhoeae: evidence for gene conversion. Cell 44, 107-115.

Haas, R., Schwarz, H. \& Meyer, T. F. (1987). Release of soluble pilin antigen coupled with gene conversion in Neisseria gonorrhoeae. Proc Natl Acad Sci U S A 84, 9079-9083.
Haas, R., Veit, S. \& Meyer, T. F. (1992). Silent pilin genes of Neisseria gonorrhoeae MS11 and the occurrence of related hypervariant sequences among other gonococcal isolates. Mol Microbiol 6, 197-208.

Hagblom, P., Segal, E., Billyard, E. \& So, M. (1985). Intragenic recombination leads to pilus antigenic variation in Neisseria gonorrhoeae. Nature 315, 156-158.

Howell-Adams, B. \& Seifert, H. S. (2000). Molecular models accounting for the gene conversion reactions mediating gonococcal pilin antigenic variation. Mol Microbiol 37, 1146-1158.

Jennings, M. P., Virji, M., Evans, D., Foster, V., Srikhanta, Y. N., Steeghs, L., van der Ley, P. \& Moxon, E. R. (1998). Identification of a novel gene involved in pilin glycosylation in Neisseria meningitidis. Mol Microbiol 29, 975-984.

Jerse, A. E., Cohen, M. S., Drown, P. M., Whicker, L. G., Isbey, S. F., Seifert, H. S. \& Cannon, J. G. (1994). Multiple gonococcal opacity proteins are expressed during experimental urethral infection in the male. J Exp Med 179, 911-920.

Jonsson, A.-B., Ilver, D., Falk, P., Pepose, J. \& Normark, S. (1994). Sequence changes in the pilus subunit lead to tropism variation of Neisseria gonorrhoeae to human tissue. Mol Microbiol 13, 403-416.

Källström, H., Liszewski, M. K., Atkinson, J. P. \& Jonsson, A.-B. (1997). Membrane cofactor protein (MCP or CD46) is a cellular pilus receptor for pathogenic Neisseria. Mol Microbiol 25, 639-647.

Kellogg, D. S., Peacock, W. L., Deacon, W. E., Brown, L. \& Pirkle, C. I. (1963). Neisseria gonorrhoeae. 1. Virulence genetically linked to clonal variation. J Bacteriol 85, 1274-1279.

Koomey, M., Gotschlich, E. C., Robbins, K., Bergstrom, S. \& Swanson, J. (1987). Effects of recA mutations on pilus antigenic variation and phase transitions in Neisseria gonorrhoeae. Genetics 117, 391-398.

Lambden, P. R., Robertson, J. N. \& Watt, P. J. (1980). Biological properties of two distinct pilus types produced by isogenic variants of Neisseria gonorrhoeae P9. J Bacteriol 141, 393-396.

Long, C. D., Madraswala, R. N. \& Seifert, H. S. (1998). Comparisons between colony phase variation of Neisseria gonorrhoeae FA1090 and pilus, pilin, and S-pilin expression. Infect Immun 66, 1918-1927.

Marceau, M. \& Nassif, X. (1999). Role of glycosylation at Ser63 in production of soluble pilin in pathogenic Neisseria. J Bacteriol 181, 656-661.

Marceau, M., Beretti, J. L. \& Nassif, X. (1995). High adhesiveness of encapsulated Neisseria meningitidis to epithelial cells is associated with the formation of bundles of pili. Mol Microbiol 17, 855-863.

Marceau, M., Forest, K., Beretti, J. L., Tainer, J. \& Nassif, X. (1998). Consequences of the loss of O-linked glycosylation of meningococcal type IV pilin on piliation and pilus-mediated adhesion. Mol Microbiol 27, 705-715.

Mehr, I. J. \& Seifert, H. S. (1998). Differential roles of homologous recombination pathways in Neisseria gonorrhoeae pilin antigenic variation, DNA transformation and DNA repair. Mol Microbiol 30, 697-710.

Meyer, T. F., Billyard, E., Haas, R., Storzbach, S. \& So, M. (1984). Pilus genes of Neisseria gonorrhoeae: chromosomal organization and DNA sequence. Proc Natl Acad Sci U S A 81, 6110-6114.

Nassif, X. \& So, M. (1995). Interaction of pathogenic neisseriae with nonphagocytic cells. Clin Microbiol Rev 8, 376-388. 
Nassif, X., Lowry, J., Stenberg, P., O’Gaora, P., Ganji, A. \& So, M. (1993). Antigenic variation of pilin regulates adhesion of Neisseria meningitidis to human epithelial cells. Mol Microbiol 8, 719-725.

Nassif, X., Pujol, C., Morand, P. \& Eugène, E. (1999). Interactions of pathogenic Neisseria with host cells. Is it possible to assemble the puzzle? Mol Microbiol 32, 1124-1132.

Rudel, T., Scheuerpflug, I. \& Meyer, T. F. (1995). Neisseria PilC protein identified as type-4 pilus tip-located adhesin. Nature 373, $357-362$.

Sambrook, J., Fritsch, E. F. \& Maniatis, T. (1989). Molecular Cloning: a Laboratory Manual, 2nd edn. Cold Spring Harbor, NY: Cold Spring Harbor Laboratory.

Seifert, H. S. (1996). Questions about gonococcal pilus phase and antigenic variation. Mol Microbiol 21, 433-440.

Seifert, H. S., Wright, C. S., Jerse, A. E., Cohen, M. S. \& Cannon, J. G. (1994). Multiple gonococcal pilin antigenic variants are produced during experimental human infections. J Clin Invest $\mathbf{9 3 ,}$ 2744-2749.

Smith, H. O. \& Birnstiel, M. L. (1976). A simple method for DNA restriction site mapping. Nucleic Acids Res 3, 2387-2398.

Swanson, J., Robbins, K., Barrera, O., Corwin, D., Boslego, J., Ciak, J., Blake, M. \& Koomey, J. M. (1987). Gonococcal pilin variants in experimental gonorrhea. J Exp Med 165, 1344-1357.
Virji, M., Saunders, J. R., Sims, G., Makepeace, K., Maskell, D. \& Ferguson, D. J. (1993). Pilus-facilitated adherence of Neisseria meningitidis to human epithelial and endothelial cells: modulation of adherence phenotype occurs concurrently with changes in primary amino acid sequence and the glycosylation status of pilin. Mol Microbiol 10, 1013-1028.

Wainwright, L. A., Pritchard, K. H. \& Seifert, H. S. (1994). A conserved DNA sequence is required for efficient gonococcal pilin antigenic variation. Mol Microbiol 13, 75-87.

Wainwright, L. A., Frangipane, J. V. \& Seifert, H. S. (1997). Analysis of protein binding to the Sma/Cla DNA repeat in pathogenic neisseriae. Nucleic Acids Res 25, 1362-1368.

Wright, C. J., Jerse, A. E., Cohen, M. S., Cannon, J. G. \& Seifert, H. S. (1994). Nonrepresentative PCR amplification of variable gene sequences in clinical specimens containing dilute, complex mixtures of microorganisms. J Clin Microbiol 32, 464-468.

Zak, K., Diaz, J. L., Jackson, D. \& Heckels, J. E. (1984). Antigenic variation during infection with Neisseria gonorrhoeae: detection of antibodies to surface proteins in sera of patients with gonorrhea. J Infect Dis 149, 166-174.

Received 10 October 2000; revised 4 January 2001; accepted 5 January 2001. 